[Supporting Information]

\title{
Multi-Nuclear Lanthanide-Implanted Tetrameric Dawson-type Phosphotungstates with Switchable Luminescence Behaviors Induced by Fast Photochromism
}

Bing Yan, Rongchang Liang, Kangting Zheng, Rui Li, Pengtao Ma, * Jingping Wang and Jingyang Niu *

Henan Key Laboratory of Polyoxometalate Chemistry, Institute of Molecular and Crystal Engineering, College of Chemistry and Chemical Engineering, Henan University, Kaifeng 475004, Henan (P.R. China)

\section{CONTENTS}

Section 1 Experimental Section

Section 2 Supplementary Structural Figures and Tables

Section 3 Additional Measurements 


\section{Section 1 Experimental Section}

\section{Materials and physical methods}

All chemicals were commercially purchased and used without any further purification. The precursor $\mathrm{K}_{14}\left[\mathrm{P}_{2} \mathrm{~W}_{15} \mathrm{O}_{56}\right] \cdot 24 \mathrm{H}_{2} \mathrm{O}$ was prepared on account of the document and confirmed by IR spectrum. C, $\mathrm{H}$ and $\mathrm{N}$ elemental analyses were conducted on a Perkin-Elmer 2400-II CHNS/O analyzer. The contents of $\mathrm{P}, \mathrm{W}, \mathrm{Sm}, \mathrm{Eu}$, and Gd elements were measured by inductively coupled plasma atomic emission spectrometry (ICP-AES) on a Perkin-Elmer Optima 2000 ICP-AES spectrometer. A Bruker D8 ADVANCE apparatus with $\mathrm{Cu}$ Karadiation at $293 \mathrm{~K}$ gave birth to the experimental PXRD patterns. IR spectra were performed using a Bruker VERTEX-70 spectrometer using $\mathrm{KBr}$ pellets in the region of $400-4000 \mathrm{~cm}^{-1}$. TGA cures were recorded from room $25^{\circ} \mathrm{C}$ to $800{ }^{\circ} \mathrm{C}$ with a heating rate of $10^{\circ} \mathrm{C} \mathrm{min}^{-1}$ in flowing $\mathrm{N}_{2}$ atmosphere on a NETZSCH STA 449 F5 Jupiter thermal analyzer. Diffuse reflectance spectra were collected at room temperature on a finely ground sample with a HITACHI U-4500 UV-Vis-NIR spectrometer equipped with a $60 \mathrm{~mm}$ diameter integrating sphere. Photoluminescence emission and excitation spectra were measured on a FLS 980 Edinburgh Analytical Instrument under a $450 \mathrm{~W}$ xenon lamp. Decay curves and time resolved emission spectra (TRES) were obtained under the excitation of a $\mu \mathrm{F} 900 \mathrm{H}$ high-energy microsecond flash lamp.

\section{Synthesis}

$\mathrm{SmCl}_{3} \cdot 6 \mathrm{H}_{2} \mathrm{O}(0.366 \mathrm{~g}, 1 \mathrm{mmol})$ was dissolved in water $(40 \mathrm{~mL})$, followed by the solid addition of benzoic acid $(0.120 \mathrm{~g}, 0.100 \mathrm{mmol})$ and $\mathrm{K}_{14}\left[\mathrm{P}_{2} \mathrm{~W}_{15} \mathrm{O}_{56}\right] \cdot 24 \mathrm{H}_{2} \mathrm{O}(0.110 \mathrm{~g}, 0.25 \mathrm{mmol})$ were added. The mixed solution stirred constantly to obtain a clear solution. Finally, the $\mathrm{pH}$ value of the clear solution was adjusted to $3.2-3.5$ by $3 \mathrm{~mol} \cdot \mathrm{L}^{-1} \mathrm{NaOH}$ solution. The resulting solution was heated to $80^{\circ} \mathrm{C}$ for about $2 \mathrm{~h}$, and then tetramethylammonium chloride (TMACl) $(0.220 \mathrm{~g}$, $2.000 \mathrm{mmol}$ ) was added and stirred for about $30 \mathrm{~min}$. The resulting solution was cooled and filtered to evaporate at room temperature. The clear filtrate was left for about two days to gain colorless block crystals. Yield: $20.7 \%\left(0.030 \mathrm{~g}\right.$, based on $\left.\mathrm{SmCl}_{3} \cdot 6 \mathrm{H}_{2} \mathrm{O}\right)$. Elemental analysis (\%) calcd for (1): C, 2.20; H, 1.55; N, 0.28; P, 1.24; Sm, 3.02; W, 62.52. Found: C, 2.24; H, 1.62; N, $0.24 ; \mathrm{P}, 1.31 ; \mathrm{Sm}, 3.12 ; \mathrm{W}, 60.76$.

The synthesis of 2 was similar to that of $\mathbf{1}$ except that $\mathrm{SmCl}_{3} \cdot 6 \mathrm{H}_{2} \mathrm{O}$ was replaced by $\mathrm{EuCl}_{3} \cdot 6 \mathrm{H}_{2} \mathrm{O}$. Finally, colorless block crystals were collected after about three days. Yield: $32.4 \%$ (0.036 g, based on $\mathrm{EuCl}_{3} \cdot 6 \mathrm{H}_{2} \mathrm{O}$ ). Elemental analysis (\%) calcd for (2): C, 2.24; H, 1.55; N, 0.28; P, 1.24; Eu, 3.02; W, 62.52. Found: C 2.40; H, 1.71; N, 0.23; P, 1.17; Eu, 3.09; W, 61.13.

The synthesis of 3 was similar to that of 1 except that $\mathrm{SmCl}_{3} \cdot 6 \mathrm{H}_{2} \mathrm{O}$ was replaced by $\mathrm{GdCl}_{3} \cdot 6 \mathrm{H}_{2} \mathrm{O}$. Finally, colorless block crystals were collected after about three days. Yield: $30.6 \%$ $\left(0.034 \mathrm{~g}\right.$, based on $\left.\mathrm{GdCl}_{3} \cdot 6 \mathrm{H}_{2} \mathrm{O}\right)$. Elemental analysis (\%) calcd for (3): C, $2.17 ; \mathrm{H}, 1.55 ; \mathrm{N}, 0.28 ; \mathrm{P}$, 1.24; Gd, 3.38; W, 62.43. Found: C, 2.23; H, 1.70; N, 0.23; P, 1.26; Gd, 3.17; W, 61.67. 


\section{X-ray Crystallography}

The suitable crystals of 1-3 were selected and the structural measurements were performed at $150 \mathrm{~K}$ on a Bruker D8 VENTURE PHOTON II diffractometer using graphite-monochromated Mo $\mathrm{K} \alpha$ radiation $(\lambda=0.71073 \AA)$. After data collection, dates reduction, including a correction for routine Lorentz and polarization, was performed by an applied multi-scan absorption correction SADABS program. The crystal structures were solved by the direct method and refined by full matrix least-squares on all $F^{2}$ data using the SHELXL-2018/3 program package. In the final refinement, the $\mathrm{W}, \mathrm{Ln}, \mathrm{P}$ and $\mathrm{Cl}$ atoms of $\mathbf{1 - 3}$ were refined anisotropically, other light atoms $(\mathrm{C}, \mathrm{N}$ and $\mathrm{O}$ ) were refined isotropically. Moreover, the partial lattice water molecules were located by Fourier map; the remaining lattice water molecules were determined by TGA results (Figure S9). The organic hydrogen atoms were generated geometrically, all $\mathrm{H}$ atoms on water molecules were directly included in the molecular formula.

\section{Section 2 Supplementary Structural Figures and Tables}

Table S1. Crystallographic data and structure refinement parameters for $\mathbf{1}$.

\begin{tabular}{|c|c|c|c|}
\hline & 1 & 2 & 3 \\
\hline \multirow{2}{*}{ Empirical formula } & $\mathrm{C}_{38} \mathrm{H}_{82} \mathrm{Cl}_{2} \mathrm{~N}_{6} \mathrm{O}_{290} \mathrm{Sm}_{6}$ & $\mathrm{C}_{38} \mathrm{H}_{82} \mathrm{Cl}_{2} \mathrm{Eu}_{6} \mathrm{~N}_{6} \mathrm{O}_{294}$ & $\mathrm{C}_{38} \mathrm{H}_{82} \mathrm{Cl}_{2} \mathrm{Gd}_{6} \mathrm{~N}_{6} \mathrm{O}_{288}$ \\
\hline & $\mathrm{P}_{8} \mathrm{~W}_{68}$ & $\mathrm{P}_{8} \mathrm{~W}_{68}$ & $\mathrm{P}_{8} \mathrm{~W}_{68}$ \\
\hline Formula weight & 18985.65 & 19059.31 & 18995.05 \\
\hline Temperature / K & 150 & 150 & 150.0 \\
\hline Crystal system & monoclinic & monoclinic & monoclinic \\
\hline Space group & $\mathrm{P} 2_{1} / \mathrm{c}$ & $\mathrm{P} 2_{1} / \mathrm{c}$ & $\mathrm{P} 2{ }_{1} / \mathrm{c}$ \\
\hline$a[\AA]$ & $19.0384(11)$ & $19.1147(5)$ & $18.9874(16)$ \\
\hline$b[\AA]$ & $51.088(2)$ & $50.9672(12)$ & $50.597(4)$ \\
\hline$c[\AA]$ & $23.6490(11)$ & $23.6192(6)$ & $23.5181(17)$ \\
\hline$\alpha\left[^{\circ}\right]$ & 90 & 90 & 90 \\
\hline$B\left[^{\circ}\right]$ & $101.320(2)$ & $101.2850(10)$ & $101.125(2)$ \\
\hline$\gamma\left[^{\circ}\right]$ & 90 & 90 & 90 \\
\hline$V\left[\AA^{3}\right]$ & $22554(2)$ & $22565.5(10)$ & $22169(3)$ \\
\hline$Z$ & 2 & 2 & 2 \\
\hline$\rho_{\text {calcd }}\left[\mathrm{g} \mathrm{cm}^{-3}\right]$ & 2.796 & 2.805 & 2.846 \\
\hline$\mu\left[\mathrm{mm}^{-1}\right]$ & 18.142 & 18.187 & 18.559 \\
\hline$F(000)$ & 16460.0 & 16536.0 & 16452.0 \\
\hline \multirow{5}{*}{ Index ranges } & $-22 \leq \mathrm{h} \leq 22$ & & $-18 \leq h$ \\
\hline & $-60 \leq \mathrm{k} \leq 60$ & $-22 \leq \mathrm{h} \leq 20$ & $-18 \leq n \leq 22$ \\
\hline & $-28 \leq 1 \leq 28$ & $-60 \leq \mathrm{k} \leq 57$ & $-60 \leq \mathrm{k} \leq 59$ \\
\hline & & $-26 \leq 1 \leq 28$ & \\
\hline & & & $-28 \leq 1 \leq 27$ \\
\hline Reflections collected & 169479 & 107681 & 120520 \\
\hline Independent reflections & $40018\left[\mathrm{R}_{\mathrm{int}}=0.0525\right]$ & $39912\left[\mathrm{R}_{\mathrm{int}}=0.0344\right]$ & $39331\left[\mathrm{R}_{\mathrm{int}}=0.0765\right]$ \\
\hline
\end{tabular}




\begin{tabular}{llll} 
data/restraints/parameters & $40018 / 25 / 1041$ & $39912 / 37 / 1064$ & $39326 / 25 / 1038$ \\
Goodness-of-fit on $F^{2}$ & 1.019 & 1.027 & 1.033 \\
$R_{1}, \mathrm{w} R_{2}[I>2 \sigma(I)]$ & $0.0918,0.2455$ & $0.0628,0.1673$ & $0.0982,0.2425$ \\
$R_{1}, \mathrm{w} R_{2}$ [all data] & $0.0980,0.2498$ & $0.0662,0.1694$ & $0.1201,0.2566$ \\
\hline
\end{tabular}

Table S2. Possible geometries of eight coordinated metal centers

\begin{tabular}{lll}
\hline geometry & point group & Polyhedron \\
\hline OP-8 & $D_{8 h}$ & Octagon \\
HPY-8 & $C_{7 v}$ & Heptagonal pyramid \\
HBPY-8 & $D_{6 h}$ & Hexagonal bipyramid \\
CU-8 & $O_{h}$ & Cube \\
SAPR-8 & $D_{4 d}$ & Square antiprism \\
TDD-8 & $D_{2 d}$ & Triangular dodecahedron \\
JGBF-8 & $D_{2 d}$ & Johnson gyrobifastigium (J26) \\
JETBPY-8 & $D_{3 h}$ & Johnson elongated triangular bipyramid (J14) \\
JBTPR-8 & $C_{2 v}$ & Biaugmented trigonal prism (J50) \\
BTPR-8 & $C_{2 v}$ & Biaugmented trigonal prism \\
JSD-8 & $D_{2 d}$ & Snub diphenoid (J84) \\
TT-8 & $T_{d}$ & Triakis tetrahedron \\
ETBPY-8 & $D_{3 h}$ & Elongated trigonal bipyramid \\
\hline
\end{tabular}

Table S3. Possible geometries of eight coordinated metal centers

\begin{tabular}{llll}
\hline & Sm1 & Sm2 & Sm3 \\
\hline OP-8 & 29.279 & 27.92 & 32.451 \\
HPY-8 & 22.353 & 23.098 & 23.724 \\
HBPY-8 & 16.83 & 17.179 & 16.239 \\
CU-8 & 10.478 & 10.602 & 10.357 \\
SAPR-8 & 0.304 & 0.167 & 2.958 \\
TDD-8 & 2.217 & 2.58 & 0.958 \\
JGBF-8 & 16.3 & 16.364 & 13.929 \\
JETBPY-8 & 28.143 & 28.007 & 28.91 \\
JBTPR-8 & 2.349 & 2.852 & 2.465 \\
BTPR-8 & 1.657 & 2.287 & 1.426 \\
JSD-8 & 4.96 & 5.307 & 3.663 \\
TT-8 & 11.207 & 11.204 & 11.129 \\
ETBPY-8 & 23.36 & 23.592 & 23.999 \\
\hline
\end{tabular}

Table S4. The optical band gap values of 1-3

\begin{tabular}{|l|l|l|}
\hline Compound & $\begin{array}{l}\text { optical band gap values before } \\
\text { irradiation }\end{array}$ & $\begin{array}{l}\text { optical band gap values after } \\
\text { irradiation }\end{array}$ \\
\hline
\end{tabular}




\begin{tabular}{|l|l|l|}
\hline $\mathbf{1}$ & $2.96 \mathrm{eV}$ & $2.79 \mathrm{eV}$ \\
\hline $\mathbf{2}$ & $2.97 \mathrm{eV}$ & $2.86 \mathrm{eV}$ \\
\hline $\mathbf{3}$ & $2.87 \mathrm{eV}$ & $2.76 \mathrm{eV}$ \\
\hline
\end{tabular}

Table S5. The photochromic speed of currently reported photochromic polyoxotungstates

\begin{tabular}{|l|l|}
\hline $\mathrm{T}_{1 / 2}$ & Refs \\
\hline $6.32 \mathrm{~min}, 21.28 \mathrm{~min}$ and $26.32 \mathrm{~min}$ & $2 \mathrm{~b}$ \\
\hline $5.4 \mathrm{~min}$ and $1.2 \mathrm{~min}$ & $18 \mathrm{a}$ \\
\hline $1.85 \mathrm{~min}$ & $18 \mathrm{~d}$ \\
\hline $1.168 \mathrm{~min}, 0.721 \mathrm{~min}, 0.687 \mathrm{~min}$ & This work \\
\hline
\end{tabular}

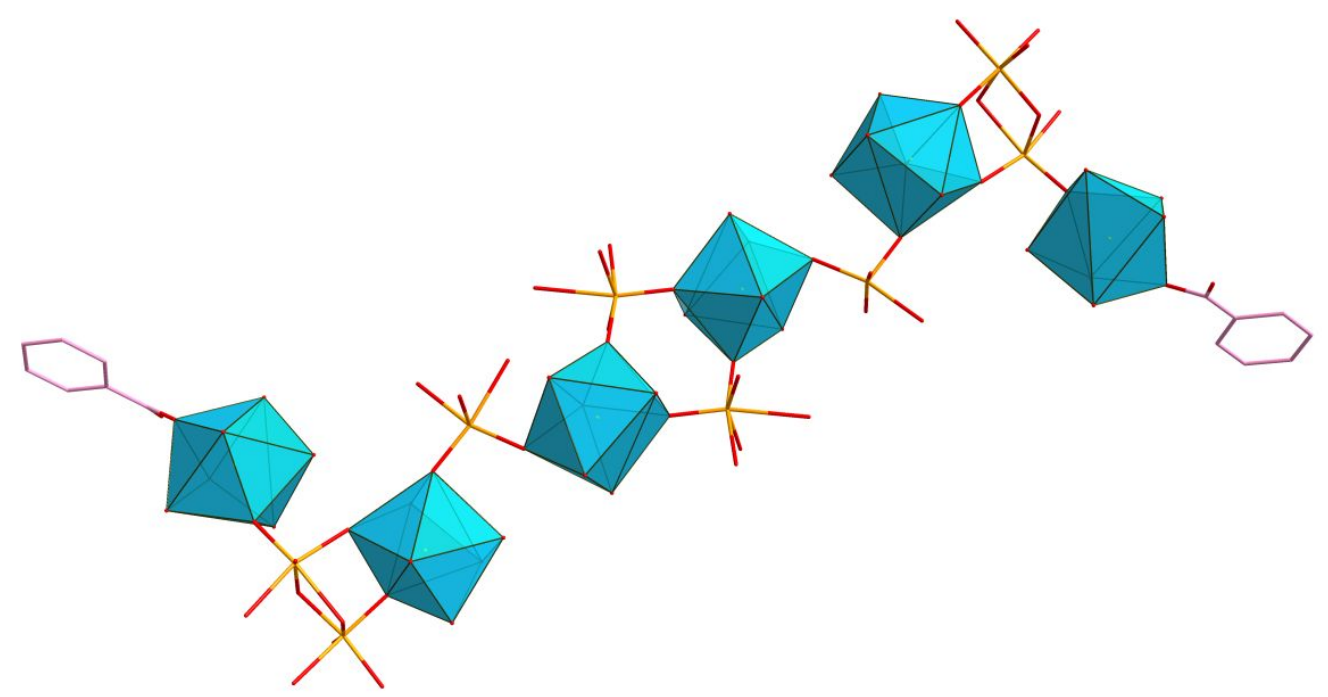

Figure S1. The sinusoidal $\mathrm{Sm}-\mathrm{O}$ cluster.

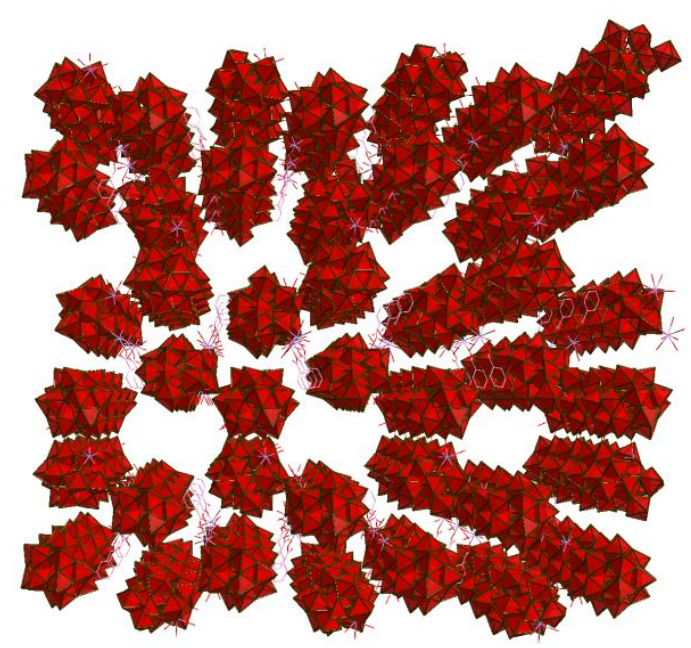


Figure S2. The packing arrangement.

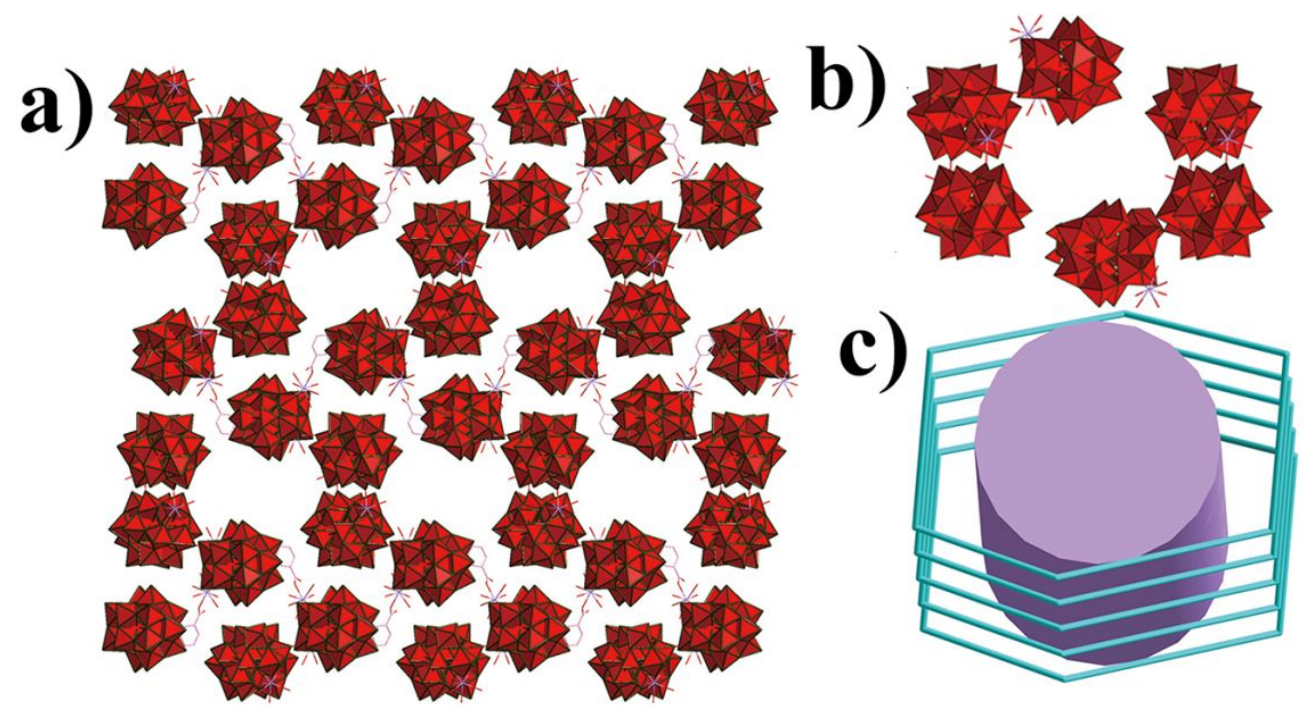

Figure S3. a) The packing arrangement of $\mathbf{1}$ along the $a$ axis. (b) Basic building block of the packing arrangement of $\mathbf{1}$ along the $a$ axis. (c) The 1-D channels of the packing arrangement of $\mathbf{1}$ along the $a$ axis

a)
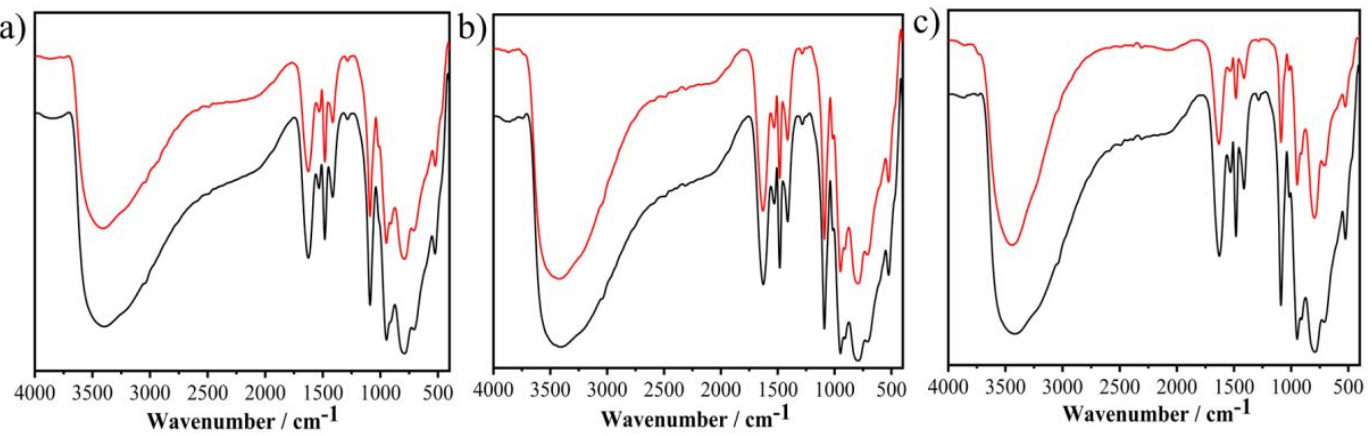

Figure S4. IR spectra of the samples before (black) and after irradiation (red) for a) 1, b) 2 and c)

3.

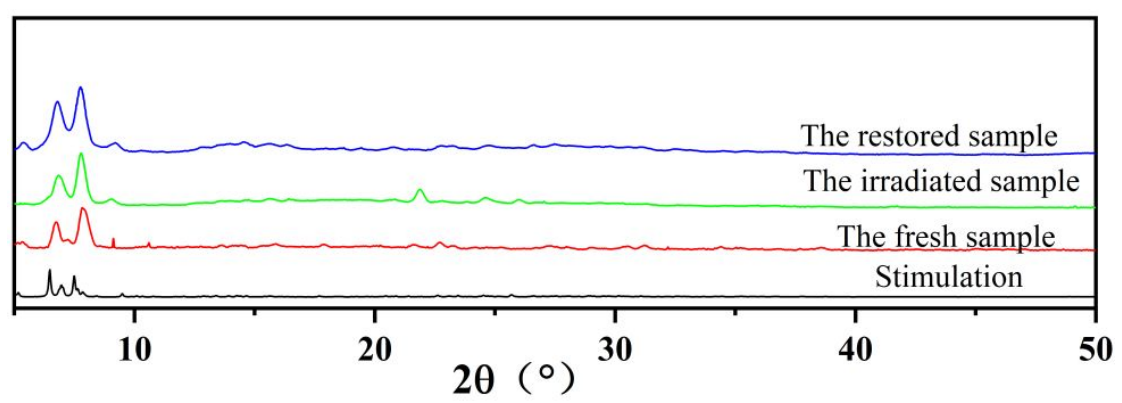

Figure S5. The PXRD of 1. 


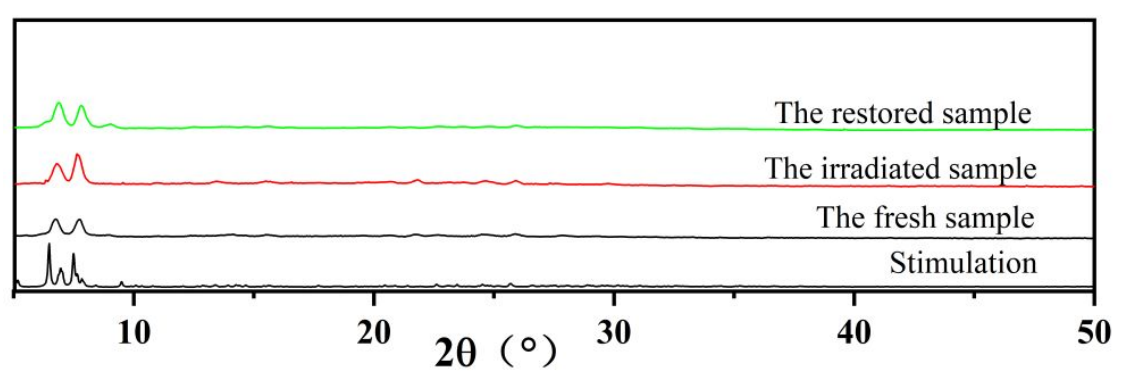

Figure S6. The PXRD of 2.

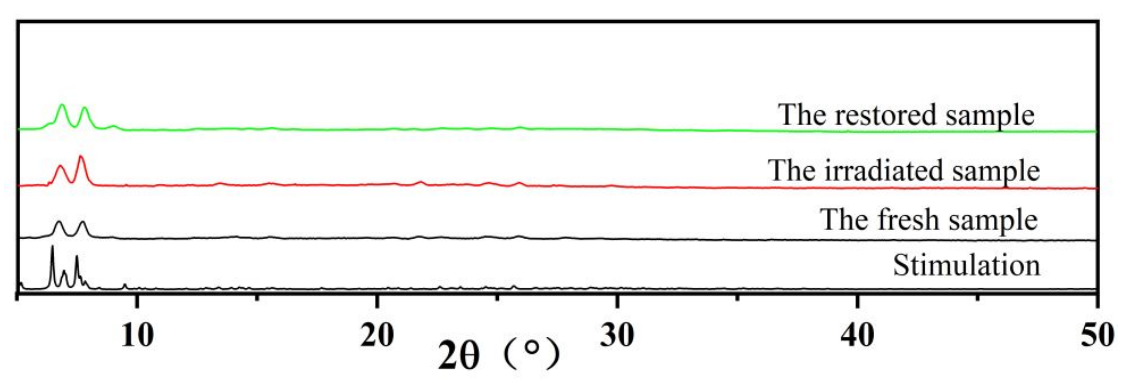

Figure S7. The PXRD of 3.

a)

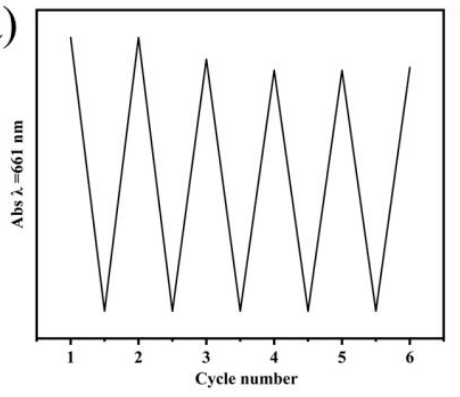

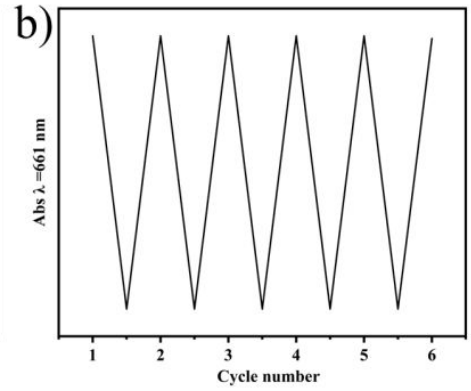

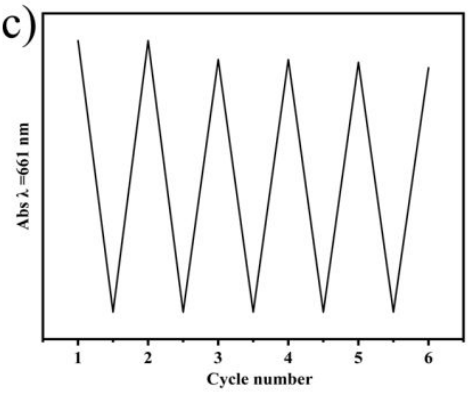

Figure S8. The values of absorption band at $\sim 661 \mathrm{~nm}$ of every cycle, 1 a), 2 b) and 3c)
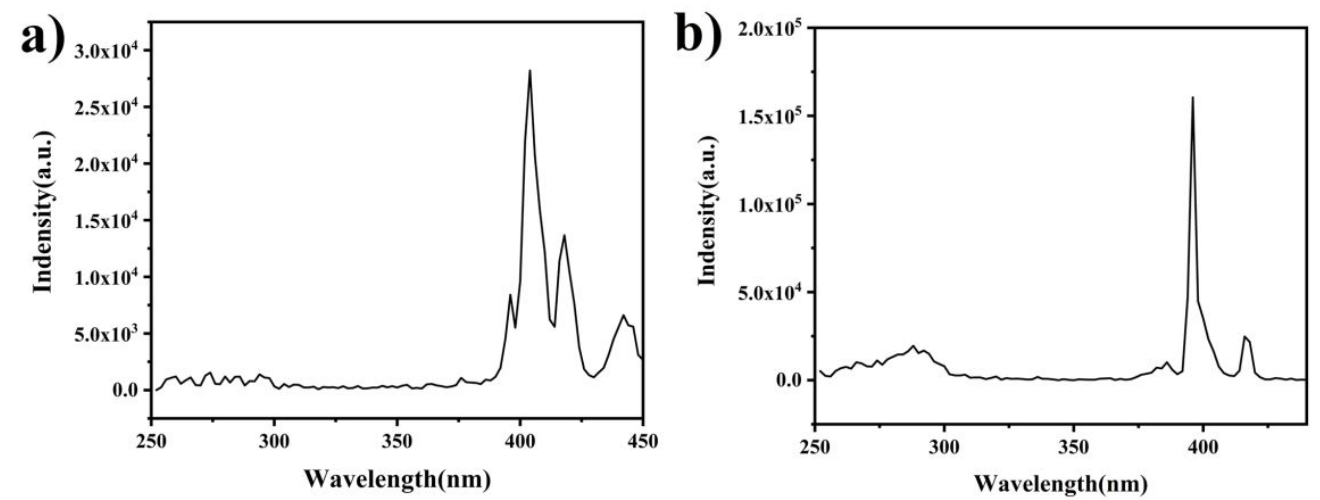

Figure S9. The excitation spectra of $\mathbf{1}$ a) and $\mathbf{2}$ b) in the solid state at room temperature. 


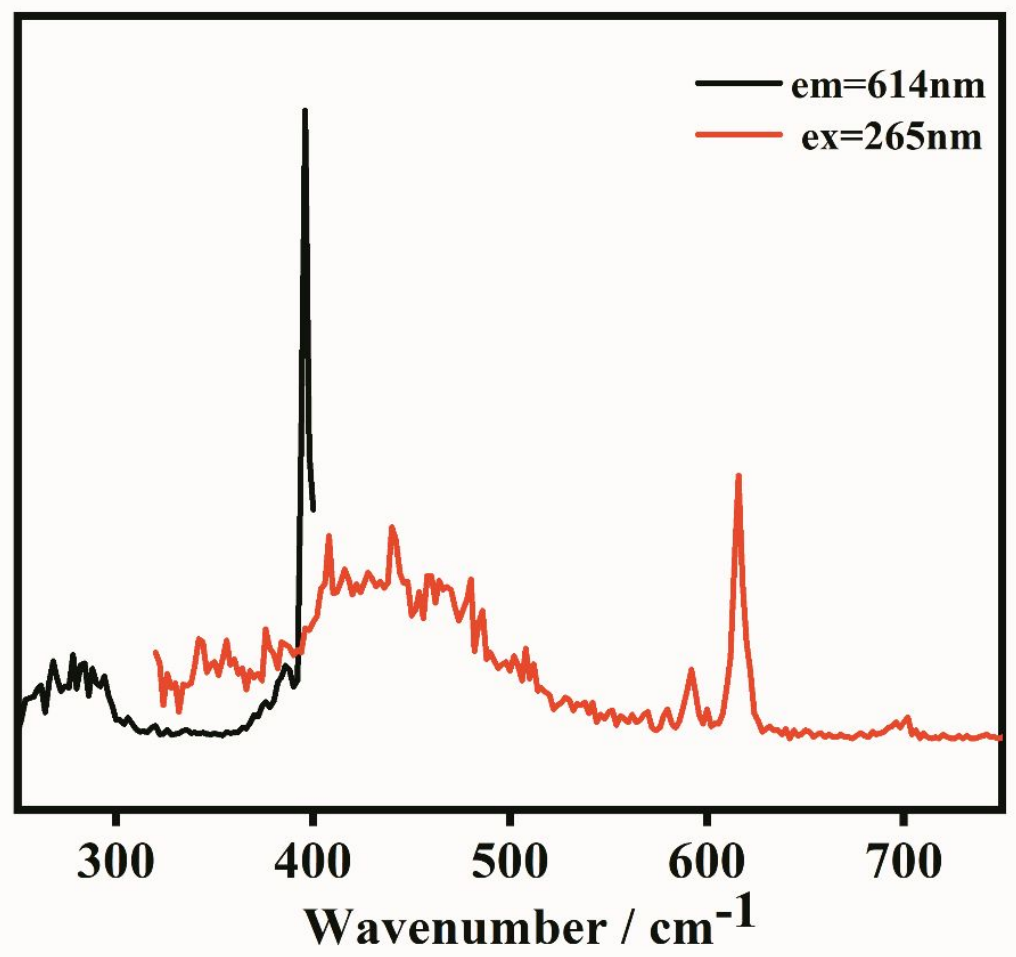

Figure S10. Luminescent spectra for 2 in 350-750 nm under $265 \mathrm{~nm}$ excitation and the excited spectrogram for 5 in $250-420 \mathrm{~nm}$ based on supervising $614 \mathrm{~nm}$ emission.

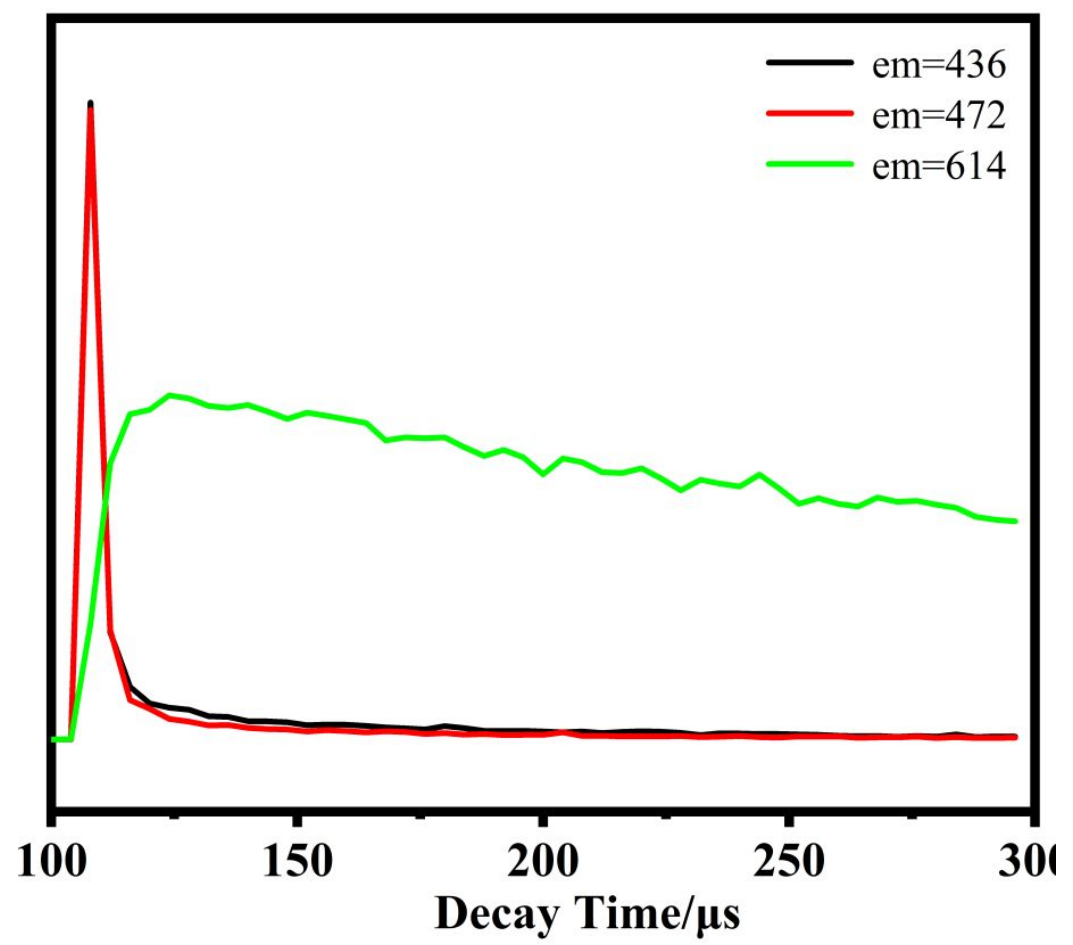

Figure S11. Intensity comparison of emitting peaks at 472, 436 and $614 \mathrm{~nm}$ with decay time. 


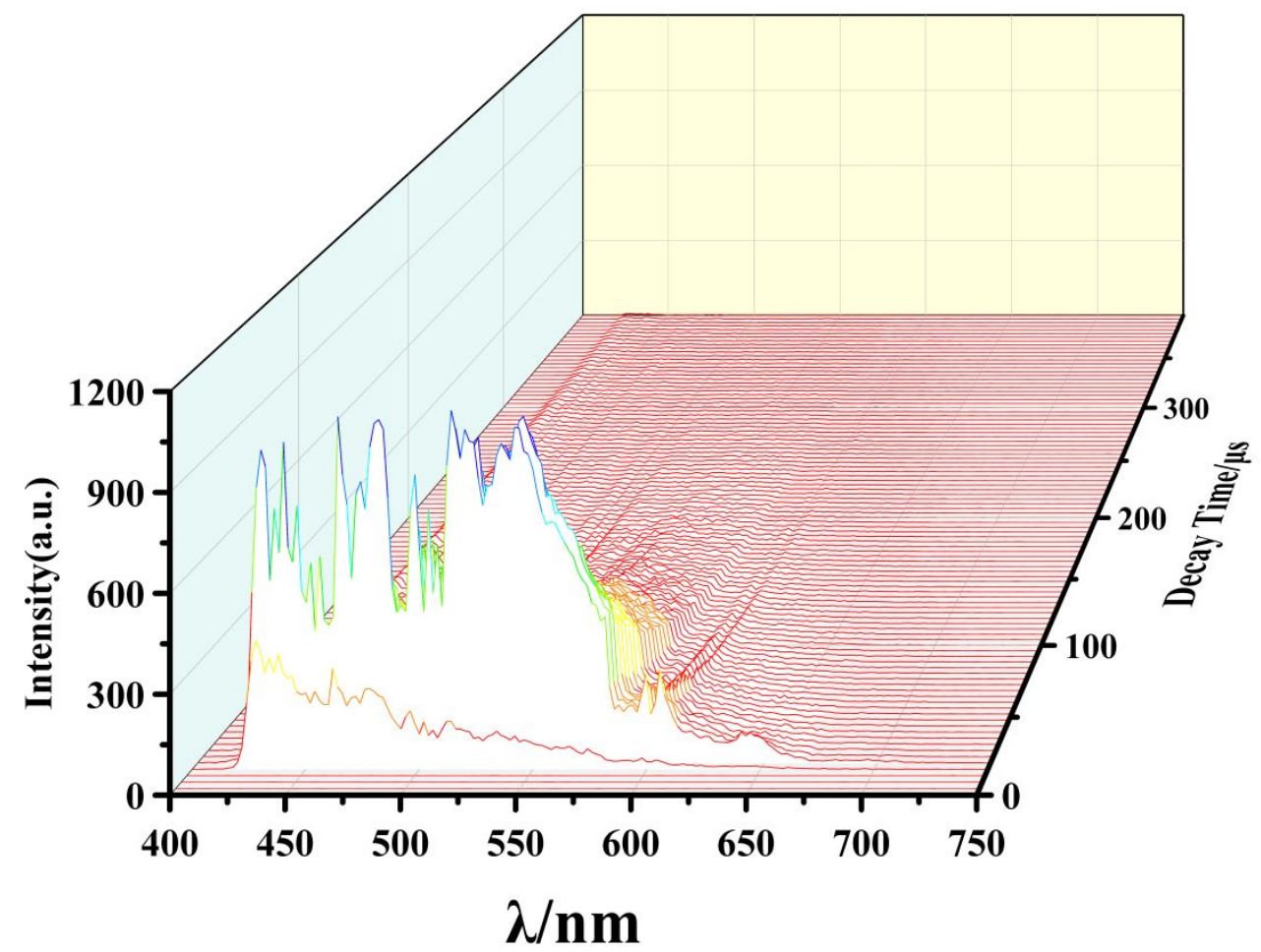

Figure S12. TRES of 1.
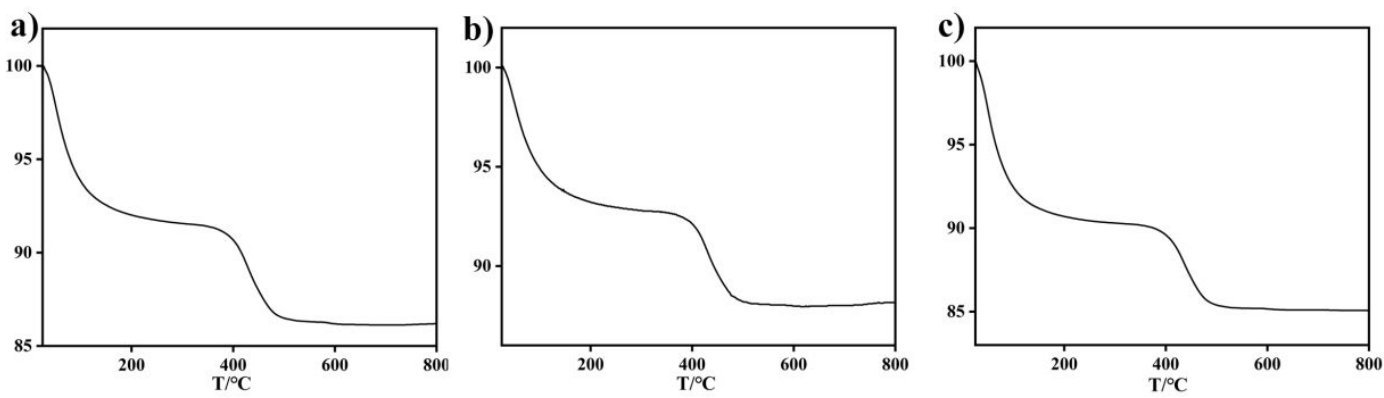

Figure S13. TGA curve of $\mathbf{1}-\mathbf{3}$ under nitrogen gas atmosphere with the heating rate of 10 ${ }^{\circ} \mathrm{C} / \mathrm{min}$ in the range of $25-800^{\circ} \mathrm{C}$. 